\title{
Direct observation of melting in a two-dimensional superconducting vortex lattice
}

\author{
I. Guillamón ${ }^{1}$, H. Suderow ${ }^{1 \star}$, A. Fernández-Pacheco ${ }^{2,3,4}$, J. Sesé2,4 ${ }^{2, ~ R . ~ C o ́ r d o b a ~}{ }^{2,4}$, J. M. De Teresa ${ }^{3,4}$, \\ M. R. Ibarra ${ }^{2,3,4}$ and S. Vieira ${ }^{1}$
}

\begin{abstract}
Topological defects such as dislocations are predicted to determine the two-dimensional (2D) melting transition ${ }^{1-4}$. In 2D superconducting vortex lattices, macroscopic measurements provide evidence for melting close to the transition to the normal state. However, the direct observation at the scale of individual vortices of the melting sequence has never been carried out. Here, we use scanning tunnelling spectroscopy (STS) to provide step-by-step imaging of a 2D system of vortices up to the melting transition in a $\mathbf{W}$-based superconducting thin film. We show directly the transition into an isotropic liquid below the superconducting critical temperature. Before that, we find a hexatic phase, characterized by the appearance of free dislocations, and a smectic-like phase, possibly formed through partial disclination unbinding. These results represent a significant step in the understanding of the melting of 2D systems, with an impact across several research fields, such as liquid-crystal molecules or lipids in membranes ${ }^{5-8}$.
\end{abstract}

The seminal ideas of Berezinskii, Kosterlitz and Thouless ${ }^{1,2}$, further developed by Halperin, Nelson and Young ${ }^{3,4}$, have been much discussed in connection with the melting of many different 2D hexagonal crystals. These include crystals formed from elongated rod-like entities such as superfluid vortices, lipids or liquid-crystal molecules, as well as from more isotropic constituents, such as electrons in Wigner crystals, particles or bubbles, or electronic charge-density arrangements ${ }^{9-11}$. Theoretically, it is found that the unbinding of dislocation pairs creates an intermediate phase in a 2D hexagonal crystal, named the hexatic phase, which retains six-fold orientational order but has no long-range translational order ${ }^{2-4}$. The hexatic phase, which appears between the $2 \mathrm{D}$ crystalline phase and the isotropic liquid, has been the subject of much research. Vortex lattices in amorphous transition-metal superconducting thin films are considered as an ideal system where the mechanism of the $2 \mathrm{D}$ melting process can be investigated. Until now, superconducting vortex-lattice melting in thin films has been studied through macroscopic experiments of thermal properties or of critical currents around the melting transition ${ }^{12-14}$. The transition clearly follows the theory of 2D dislocation-unbinding melting behaviour ${ }^{15-19}$. However, no local direct observation of melting has been provided. Here, we track the modifications induced by temperature on a $2 \mathrm{D}$ vortex system, from its formation up to the isotropic liquid state. On increasing the temperature, we first observe thermal depinning of vortices, which produces a more ordered hexagonal lattice. The melting process is then initiated with the appearance of free dislocations, corresponding to the hexatic phase, after which smectic-like vortex arrangements appear, before the isotropic liquid is formed.

Our sample is an amorphous W-based thin film deposited using an ion beam focused to nanometric size, with a superconducting critical temperature $T_{\mathrm{c}}=4.15 \mathrm{~K}$ at zero field and a thickness $d=200 \mathrm{~nm}$ (refs 20,21). It is an extreme type-II superconductor, with superconducting behaviour that is similar to that found in other amorphous transition-metal thin films ${ }^{12-14}$. Values for the coherence length and London penetration depth are, respectively, $\xi=6.25 \mathrm{~nm}$ and $\lambda=850 \mathrm{~nm}$ (see Supplementary Information). Macroscopic measurements of the critical current provide values between $10^{4}$ and $10^{5} \mathrm{~A} \mathrm{~cm}^{-2}$. As the magnetic field is applied perpendicular to the film surface, the longitudinal correlation length $L_{\mathrm{c}}$, which can be estimated from the critical current (see, for example, refs 12-14) and provides the length scale for vortex distortions parallel to the magnetic field, is of the order of $5 \mu \mathrm{m}$, which is much larger than the sample thickness $d$. So vortices indeed behave to a large extent as a $2 \mathrm{D}$ solid, as previously shown in other amorphous extreme type-II transition-metal superconducting thin films with similar parameters ${ }^{12-16}$.

The superconducting vortex lattice can be imaged using STS (ref. 22). As the superconducting gap closes inside the vortex cores, well-resolved vortex-lattice images can be obtained by means of tracking the Fermi-level (zero-bias) tunnelling conductance (the voltage derivative of the tunnelling current $\mathrm{d} I / \mathrm{d} V$ ), normalized to the value at voltages well above the superconducting gap, as a function of the position at the sample's surface (that is, tracking $\sigma(x, y)$, with $\sigma=(\mathrm{d} I / \mathrm{d} V(V=0 \mathrm{~V})) /(\mathrm{d} I / \mathrm{d} V(e V>>\Delta)), \Delta$ being the superconducting gap and $e$ the electronic charge). However, very stable and flat surfaces are required, with homogeneous superconducting tunnelling conductance curves at zero field. The compound $\mathrm{NbSe}_{2}$, with excellent surface properties, has provided the basic element for STS work in the vortex lattice, including pioneering experiments about its dynamic behaviour under current flow and as a function of temperature $\mathrm{e}^{23,24}$. However, the melting transition could not be observed. In previous work on extreme type-II superconducting thin films, it was necessary to evaporate a further small normal surface Au layer ${ }^{25,26}$. This strongly decreased the contrast and imaging capacities of the STS, so the vortex lattice has not been followed as a function of temperature. Other local real-space techniques have faced different resolution limitations close to melting ${ }^{27,28}$.

In contrast, in the thin films studied here, STS can be used in a wide range of temperatures and magnetic fields without a particular surface preparation ${ }^{21}$. Topographical (scanning tunnelling

\footnotetext{
${ }^{1}$ Laboratorio de Bajas Temperaturas, Departamento de Física de la Materia Condensada, Instituto de Ciencia de Materiales Nicolás Cabrera, Facultad de Ciencias, Universidad Autónoma de Madrid, E-28049 Madrid, Spain, ${ }^{2}$ Instituto de Nanociencia de Aragón, Universidad de Zaragoza, 50009 Zaragoza, Spain, ${ }^{3}$ Instituto de Ciencia de Materiales de Aragón, Universidad de Zaragoza-CSIC, Facultad de Ciencias, 50009 Zaragoza, Spain, ${ }^{4}$ Departamento de Física de la Materia Condensada, Universidad de Zaragoza, 50009 Zaragoza, Spain. *e-mail: hermann.suderow@uam.es.
} 


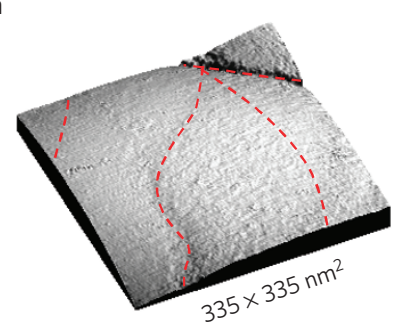

d

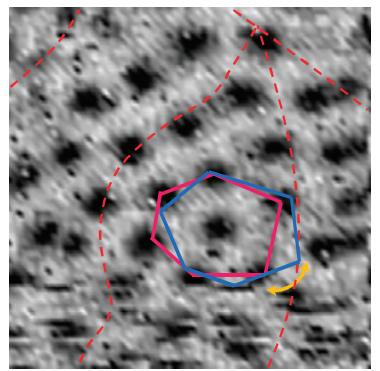

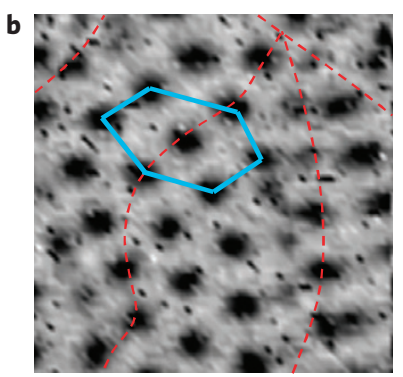

e

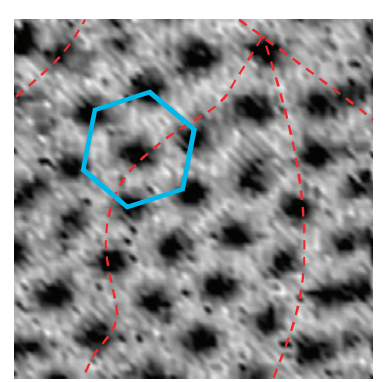

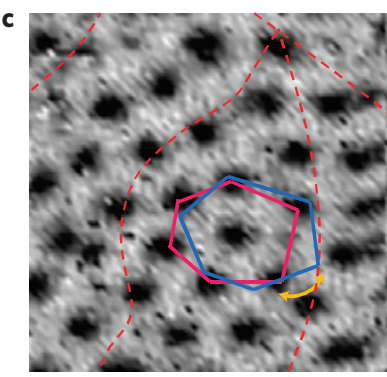

f

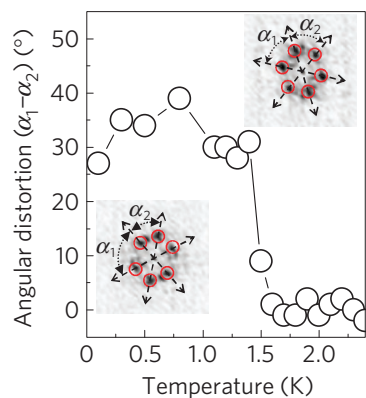

Figure 1 | Thermal depinning of the 2D vortex lattice. a, Scanning tunnelling microscopy topography, with a grey scale corresponding to height changes of $10 \mathrm{~nm}$. Surface topography features are marked in a-e as red dashed lines. b, STS vortex-lattice image after applying a magnetic field of $1 \mathrm{~T}$ at $0.1 \mathrm{~K}$. The vortices (black areas) form a hexagonal lattice, which is strongly distorted, as highlighted by the light-blue hexagon. Note that the vortices have a strong tendency to follow a near-square arrangement, imposed by the linear surface features surrounding the central part of the image. $\mathbf{c}$, When increasing the temperature, nothing changes until it reaches $1.5 \mathrm{~K}$, where the vortices start to move. The changes are subtle. The vortex lattice in the central part of the image suffers a small rotation, highlighted by magenta and blue hexagons. The magenta hexagon shows the present vortex arrangement, whereas the blue hexagon shows the same six vortices at lower temperatures. $\mathbf{d}$, The vortex arrangement switches at $1.6 \mathrm{~K}$ (as shown schematically by orange arrows) between two positions, although the temperature is fixed. e, Above $1.6 \mathrm{~K}$, the vortex lattice has found a stable position, with a more ordered triangular arrangement. f, Angular distortion of the vortex lattice as measured from an analysis of the Fourier transform of the vortex-lattice images. The angles $\alpha_{1}$ and $\alpha_{2}$, as defined in the insets, which represent the Fourier transform of the STS images at $0.1 \mathrm{~K}$ (lower left) and $1.6 \mathrm{~K}$ (upper right), strongly change at the depinning temperature.

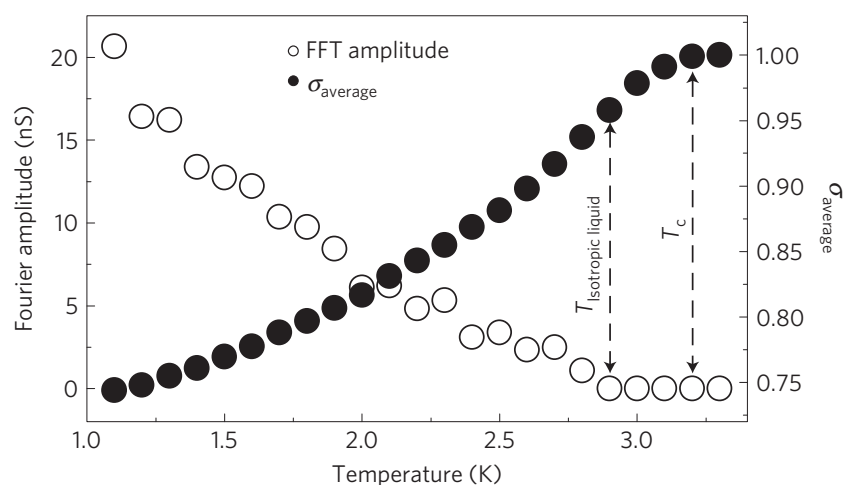

Figure 2 | Temperature-induced changes in the superconducting signal and in the contrast of the vortex-lattice STS images. Amplitude of the six-fold Bragg peaks (open circles) of the Fourier transform of the images, and the normalized zero-voltage tunnelling conductance averaged over whole images $\sigma_{\text {average }}$ (filled circles), as a function of temperature and at 2 T. $\sigma_{\text {average }}(T)$ gives the decrease in the superconducting signal in the STS images due to temperature-induced smearing of the local density of states and the decrease of the superconducting gap (see also ref. 21). The Fourier amplitude of the Bragg peaks (open circles) shows the decrease in the vortex-lattice contrast in the STS images. This has a steeper temperature dependence than $\sigma_{\text {average }}$ because of the thermal motion of the vortices. The time scale of the thermal fluctuations in the vortex positions is more than ten orders of magnitude below the time we need to make a line scan over each vortex ${ }^{15}$. Therefore, the obtained tunnelling conductance curves give the average between those inside and outside the vortices, which continuously move below the tip. Vortex-lattice contrast is lost well below $T_{c}$, signalling the transition to an isotropic liquid. microscopy) landscapes are highly uniform. Large flat areas are easily found, for which the main defects are small and smooth hills, separated by linear surface features of a few nanometres in height and around two hundred nanometres in lateral size. At zero field, tunnelling characteristics (STS) show the features expected for a simple s-wave Bardeen-Cooper-Schrieffer superconductor, with a uniform gap value $\Delta=0.66 \mathrm{meV}$ over the whole surface ${ }^{21}$. Owing to the flatness of the sample surface and the uniformity of its superconducting behaviour as probed by STS, we have been able to follow with detail the temperature-induced changes in small groups of vortices. The contrast is maximized in STS images, so that the inter-vortex superconducting state is represented as white, and the normal intra-vortex state as black ${ }^{21}$. Besides information about vortex positions, $\sigma(x, y)$ also shows directly if the sample is superconducting or normal (where $\sigma(x, y)=1$ ), which is crucial for identifying the isotropic liquid. Vortex images are made in a fixed location and at constant temperature and magnetic field (applied at low temperatures in zero-field-cooled conditions). The temperature was increased in steps of $0.1 \mathrm{~K}$, and each image was taken as quickly as possible, in most cases in about $8 \mathrm{~min}$. Several images were taken at a single temperature. More detailed information is given in the Supplementary Information. At low temperatures, typically we observe a triangular lattice that is rather disordered owing to pinning by features of the topography of the surface $^{21}$. Images have been taken at up to ten different locations, and at four magnetic fields $(1,2,3$ and $4 \mathrm{~T})$, always showing the phenomenology described by the individual regions shown below, so that the observations are representative of the whole surface.

Thermally induced depinning is shown in Fig. 1, with several representative vortex-lattice images. Here, we have searched for an area showing several surface features that act as pinning 

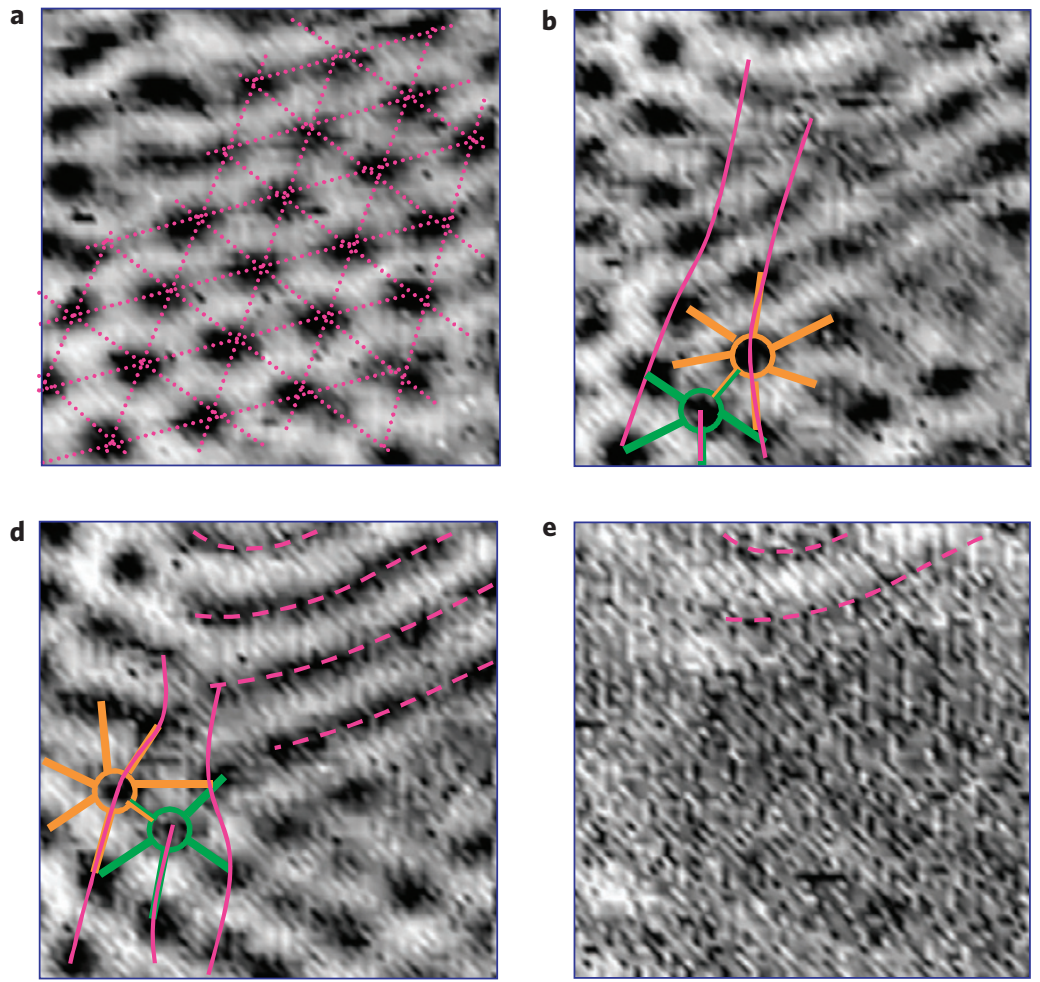
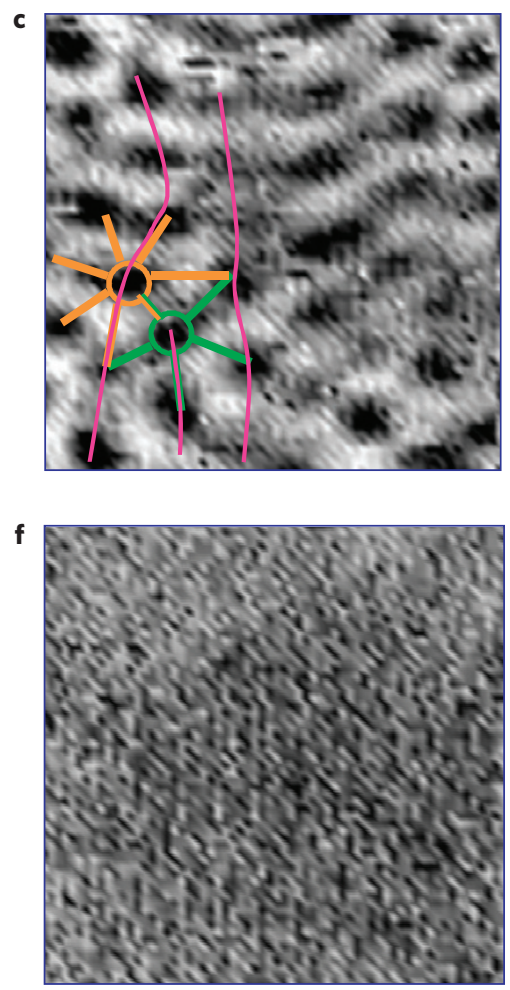

Figure 3 | Three-stage melting. a, A hexagonal lattice as imaged by STS at $1.2 \mathrm{~K}$ and a magnetic field of $2 \mathrm{~T}$ (the lateral size is $220 \mathrm{~nm}$; the black areas are individual vortices). The image is taken above the depinning temperature, so the vortex lattice is more ordered than at lower temperatures. The magenta dotted lines highlight the vortex arrangement. b. At $1.9 \mathrm{~K}$, temperature-induced changes start to become obvious. The lattice shows more disorder, and a dislocation enters the image from the bottom left. This marks the hexatic phase. The magenta lines highlight the dislocation, and the orange and green lines correspond to seven-fold- and five-fold-coordinated vortices. c, A slight temperature increase (to $2.0 \mathrm{~K}$ ) further moves the dislocation to the upper part of the image. d, At $2.1 \mathrm{~K}$, a new feature appears in the images: linear vortex arrangements (highlighted by the dashed magenta lines) in the upper right part of the image. Local contrast is lost along these lines, providing evidence for strong 1D motion. Note that the movement occurs along curved paths very similar to those produced by disclinations. Moreover, the separation between the lines corresponds to the low-temperature inter-vortex distance. This demonstrates the presence, at the local level, of a smectic-like phase. The smectic-like phase coexists with the disordered hexatic vortex lattice seen at the bottom of the image. e, At $2.8 \mathrm{~K}$, no stripes or isolated vortices are seen in the lower right part of the image. Nevertheless, the smectic-like phase is still roughly visible in the upper part. $\mathbf{f}$, At $3.0 \mathrm{~K}$, no isolated vortices or smectic-like arrangements are seen, and the superconducting gap is homogeneous, demonstrating that the vortex lattice has formed an isotropic liquid.

centres. With increasing temperature, the distorted vortex lattice does not change up to $1.5 \mathrm{~K}$. The vortices move and rearrange in the temperature interval between 1.5 and $1.6 \mathrm{~K}$. Depinning occurs essentially at the central part of the image, with thermally induced movements involving around $20-30$ vortices, and leads to a more ordered vortex lattice. The STS images and the corresponding video (see Supplementary Information) are selfexplanatory. Nevertheless, it is useful to discuss the temperature evolution of their Fourier transforms. These show the six Bragg peaks associated with the hexagonal symmetry of the vortex lattice with an angular distortion at low temperatures, which disappears abruptly at the depinning temperature of $1.5 \mathrm{~K}$ (Fig. 1f). So the lattice disorder induced through vortex pinning by features of the surface topography decreases at a temperature below that at which the melting regime appears. In particular, the size of the correlated in-plane hexagonally ordered vortex bundles, typically named $R_{\mathrm{c}}$ (ref. 16), slightly increases from around $100 \mathrm{~nm}$ up to around $200 \mathrm{~nm}$ at the depinning line (discussed below).

Let us now focus on the sequence of results approaching $T_{\mathrm{c}}$, disclosing the melting process in detail. The starting points in these experiments are hexagonal vortex bundles produced by thermal depinning. Moreover, we select flat surfaces to avoid, as far as possible, the effect of vortex pinning. The Fourier transform of the images shows six-fold Bragg peaks at $2 \pi / d$ (upper right inset of Fig. If; $d$ is the lattice constant). The average between the
Fourier amplitude of the six peaks decreases continuously with temperature (Fig. 2, at $H=2 \mathrm{~T}$ ). When the amplitude of the Bragg peaks becomes zero, we observe that the superconducting signal is not lost in the tunnelling conductance curves. $\sigma(x, y)$ is below one and uniform over the whole surface, providing evidence for a homogeneous open superconducting gap. So $\sigma(x, y)$ averaged over the whole image $\sigma_{\text {average }}$ (Fig. 2) increases continuously through the point where the Bragg peak amplitude goes to zero. At this point, the isotropic vortex liquid forms, and the vortices move below the tip, owing to thermal excitation, much faster than the imaging time, so that the $\sigma(x, y)$ obtained is the average between the results inside and outside the vortices. Finally, at $3.2 \mathrm{~K}$, the full disappearance of superconducting features in the tunnelling conductance curves, with $\sigma(x, y)$ equal to one and no longer showing any temperature dependence, marks the transition to the normal state.

Figure 3 shows six representative STS images of the melting process at $H=2 \mathrm{~T}$. At $1.2 \mathrm{~K}$, the vortex lattice shows an ordered hexagonal arrangement in most of the image (Fig. 3a). When increasing the temperature, at around $1.9 \mathrm{~K}$, we begin to observe isolated dislocations in the previously ordered lattice (Fig. 3b). They remain at the same position at a fixed temperature, but they move when the temperature is changed (Fig. 3b-d). We consider the appearance of free dislocations as the start of a hexatic phase. At a slightly higher temperature, the vortices in the upper part of the images start to be blurred, forming a partially striped image 


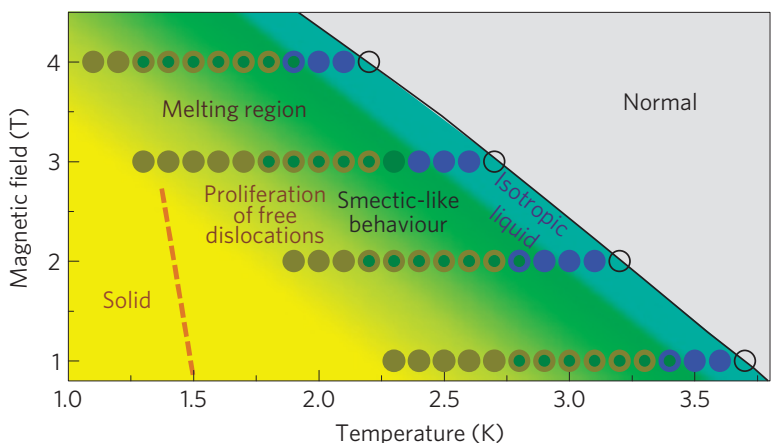

Figure 4 | Phase diagram of 2D vortex-lattice melting. The phase diagram is constructed from similar sequences as in Figs 1 and 3. The upper critical field $H_{\mathrm{c} 2}(T)$ is determined from the full loss of superconducting features (the whole temperature dependence is given in the Supplementary Information). The different behaviours that appear before, during and after the melting transition are represented by different colours: yellow, solid; yellowish green, hexatic; green, smectic-like; blue, isotropic liquid; grey, normal state. The corresponding points of the same colours give the positions where the proliferation of free dislocations (yellowish green), smectic-like behaviour (green) and liquid features (blue) are first observed at the local level in the STS images. Note that we observe a coexistence between behaviours, so that the crossover between solid, hexatic and smectic-like vortex arrangements is not well defined the macroscopic level, and we represent this by the continuously varying colour scale. The brown dashed line in the solid phase indicates the position where thermal depinning is first observed.

(Fig. 3d). The vortices have a strong 1D temperature-induced motion along these curved stripes, with the low-temperature lattice constant $d$ as the inter-stripe distance. A new smectic-like vortex arrangement can be identified by the presence of these curved stripes. In the present case (Fig. 3), a disclination may have been formed with the centre close to the scanned area. So the formation of a smectic phase can be associated with partial disclination unbinding. At $2.9 \mathrm{~K}$, the superconducting gap is observed over the whole image, but there are no isolated vortices or stripes over large parts of the image, providing evidence for a fully isotropic liquid, as shown at $3 \mathrm{~K}$ in Fig. $3 \mathrm{f}$. The images show homogeneous superconducting tunnelling features, with vortices freely moving owing to thermal excitation. At even higher temperatures, in the normal phase, the images are homogeneous and without any superconducting signal. We have followed similar images at very different densities, with lattice constants ranging from $d=50 \mathrm{~nm}$ at $1 \mathrm{~T}$ to $d=25 \mathrm{~nm}$ at $4 \mathrm{~T}$, and in up to ten different places on the surface, always finding similar behaviour. The appearance of free dislocations occurs around $1.5 \mathrm{~K}$, and the isotropic liquid is formed around $0.3 \mathrm{~K}$ below the transition to the normal phase, as shown in the phase diagram (Fig. 4) constructed from our data (the full $H_{\mathrm{c} 2}(T)$ curve is given in the Supplementary Information).

Let us note that the temperature where we first observe thermal depinning (dashed line in Fig. 4) is well differentiated from the melting process at low fields, but not at higher fields. This is in good agreement with refs 29 and 30, where it is shown that melting depends on the inter-vortex distance through a Lindemann criterion, whereas thermal depinning depends on $\xi$.

On the other hand, we often observe a coexistence of liquid with hexatic, as well as with smectic-like regions. Clearly, the transition happens for bundles of vortices, of lateral sizes below $200 \mathrm{~nm}$. Remarkably, the proliferation of defects has been observed in numerical simulations of the $2 \mathrm{D}$ vortex lattice aiming to explain macroscopic critical current measurements ${ }^{17}$. As noted there, the size of ordered regions in the low-temperature lattice is a crucial element to vortex mobility. Although it seems very difficult to make quantitative statements from our images showing only a small number of vortices, the disorder produced by the small surface roughness may indeed have a role in the size of the bundles, and possibly have some influence in the observed phenomena, which has yet to be worked out. On the other hand, with the appearance of smectic-like and isotropic liquid phases, the amplitude of thermal vortex motion is strong enough to produce blurred structures. Remarkably, these two phases mark the step-like loss of orientational order, which is maintained in the hexatic phase, where thermal vortex motion is not strong enough to blur isolated vortices in our STS images.

The emerging picture from our data is that, in addition to the appearance of free dislocations that signal the hexatic phase, a smectic-like phase arises from enhanced vortex mobility along characteristic curved lines formed by disclinations. The observed three-stage process could be a general mechanism of melting in $2 \mathrm{D}$, at least for solids formed by linear-like units, such as large molecules in liquid crystals and vortices in Abrikosov lattices. It would be interesting to know if this conclusion can be extended to other superfluids where $2 \mathrm{D}$ vortex-lattice conditions can be created, such as, for example, thin liquid-helium films or atoms in optical lattices.

\section{Received 20 April 2009; accepted 14 July 2009; published online} 9 August 2009

\section{References}

1. Berezinskii, V. Destruction of long-range order in one-dimensional and two-dimensional systems possessing a continuous symmetry group. II. Quantum systems. Sov. Phys. JETP 34, 610-616 (1972).

2. Kosterlitz, J. M. \& Thouless, D. J. Ordering, metastability and phase transitions in two-dimensional systems. J. Phys. C 6, 1181-1203 (1973).

3. Halperin, B. I. \& Nelson, D. R. Theory of two-dimensional melting. Phys. Rev. Lett. 41, 121-124 (1978).

4. Young, A. P. Dislocation-mediated melting in two dimensions. Phys. Rev. B 19, 2457-2484 (1979).

5. Chou, C.-F., Jin, A., Hui, S. W., Huang, C. C. \& Ho, J. T. Multiple-step melting in two-dimensional hexatic liquid-crystal films. Science 280, 1424-1426 (1998).

6. Zahn, K., Lenke, R. \& Maret, G. Two-stage melting of paramagnetic colloidal crystals in two dimensions. Phys. Rev. Lett. 82, 2721-2724 (1999).

7. Alsayed, A. M., Islam, M. F., Zhang, J., Collings, P. J. \& Yodh, A. G. Premelting at defects within bulk colloidal crystals. Science 309, 1207-1210 (2005).

8. Veatch, S., Soubias, O., Keller, S. \& Gawrisch, K. Critical fluctuations in domain-forming lipid mixtures. Proc. Natl Acad. Sci. USA 45, 17650-17655 (2007).

9. Altfeder, I. B. \& Chen, D. M. Anisotropic charge ordering on the gallium surface. Phys. Rev. Lett. 101, 136405 (2008).

10. Seshadri, R. \& Westervelt, R. M. Statistical mechanics of magnetic bubble arrays. I. Topology and thermalization. Phys. Rev. B 46, 5142-5149 (1992).

11. Schmitt, F. et al. Transient electronic structure and melting of a charge density wave in $\mathrm{TbTe}_{3}$. Science 321, 1649-1652 (2008).

12. Kes, P. \& Tsuei, C. Two-dimensional collective flux pinning, defects, and structural relaxation in amorphous superconducting films. Phys. Rev. B 28, 5126-5139 (1983).

13. Berghuis, P., van der Slot, A. L. F. \& Kes, P. H. Dislocation-mediated vortex-lattice melting in thin films of $\alpha-\mathrm{Nb}_{3} \mathrm{Ge}$. Phys. Rev. Lett. 65, 2583-2586 (1990).

14. Yazdani, A. et al. Observation of Kosterlitz-Thouless-type melting of the disordered vortex lattice in thin films of $\alpha$-MoGe. Phys. Rev. Lett. 70, 505-508 (1993).

15. Brandt, E. H. The flux-line lattice in superconductors. Rep. Prog. Phys. 58, 1465-1594 (1995).

16. Blatter, G., Feigel'man, M., Geshkenbein, V., Larkin, A. \& Vinokur, V. Vortices in high temperature superconductors. Rev. Mod. Phys. 66, 1125-1388 (1994).

17. Koshelev, A. E. \& Vinokur, V. M. Dynamic melting of the vortex lattice Phys. Rev. Lett. 73, 3580-3583 (1994).

18. Giamarchi, T. \& LeDoussal, P. Moving glass phase of driven lattices. Phys. Rev. Lett. 76, 3408-3411 (1996).

19. Moon, K., Scalettar, R. \& Zimámny, G. T. Dynamical phases of driven vortex systems. Phys. Rev. Lett. 77, 2778-2781 (1996).

20. Sadki, E., Ooi, S. \& Hirata, K. Focused-ion-beam-induced deposition of superconducting nanowires. Appl. Phys. Lett. 85, 6206-6208 (2004).

21. Guillamon, I. et al. Nanoscale superconducting properties of amorphous $\mathrm{W}$-based deposits grown with focused-ion-beam. New J. Phys. 10, 093005 (2008). 
22. Hess, H. F., Robinson, R. B., Dynes, R. C., Valles, J. M. \& Waszczak, J. V. Scanning-tunneling-microscope observation of the Abrikosov flux lattice and the density of states near and inside a fluxoid. Phys. Rev. Lett. 62, 214-216 (1989).

23. Troyanovski, A. M., Aarts, J. \& Kes, P. H. Collective and plastic vortex motion in superconductors at high flux densities. Nature 399, 665-668 (1999).

24. Troyanovski, A. M., van Hecke, M., Saha, N., Aarts, J. \& Kes, P. H. STM imaging of flux line arrangements in the peak effect regime. Phys. Rev. Lett. 89, 147006 (2002).

25. van Baarle, G. J. C., Troyanovski, A. M., Kes, P. H. \& Aarts, J. STM imaging vortex configurations in thin films of $\alpha-\mathrm{Mo}_{3} \mathrm{Ge}$ through a Au layer. Physica $\mathrm{C}$ 369, 335-338 (2002).

26. van Baarle, G. J. C., Troyanovski, A. M., Nishizaki, R., Kes, P. H. \& Aarts, J. Imaging of vortex configurations in thin films by scanning-tunneling-microscopy. Appl. Phys. Lett. 82, 1081-1083 (2003).

27. Harada, K. et al. Real-time observation of vortex lattices in a superconductor by electron microscopy. Nature 360, 51-53 (1992).

28. Harada, K. et al. Direct observation of vortex dynamics in superconducting films with regular arrays of defects. Science 274, 1167-1170 (1996).

29. Feigel'man, M. \& Vinokur, V. Thermal fluctuations of vortex lines, pinning and creep in high- $T_{\mathrm{c}}$ superconductors. Phys. Rev. B 41, 8986-8990 (1990).

30. Vinokur, V. M., Marchetti, M. C. \& Chen, L.-W. Glassy motion of elastic manifolds. Phys. Rev. Lett. 77, 1845-1848 (1996).

\section{Acknowledgements}

We acknowledge discussions with F. Guinea and A.I. Buzdin. The Laboratorio de Bajas Temperaturas is associated with the ICMM of the CSIC. This work was supported by the Spanish MICINN (Consolider Ingenio Molecular Nanoscience CSD2007-00010 program, MAT2008-06567-C02 and FIS2008-00454), the Comunidad de Madrid through program 'Science and Technology at Millikelvin', the Aragon Regional Government and NES and ECOM programs of the ESF.

\section{Author contributions}

I.G. carried out and designed the experiments, analysed data and wrote the paper. H.S. designed the experimental set-up, supervised the experiment and analysis, and wrote the paper. A.F.-P., R.C., J.S., J.M.D.T. and M.R.I. carried out the growth and characterization of the samples, carried out the critical current measurements and contributed to the manuscript text. J.M.D.T. and M.R.I. proposed to study the samples with STS. S.V. supervised the experiment and analysis, and wrote the paper.

\section{Additional information}

Supplementary information accompanies this paper on www.nature.com/naturephysics. Reprints and permissions information is available online at http://npg.nature.com/ reprintsandpermissions. Correspondence and requests for materials should be addressed to H.S. 